\title{
DE LA SENSIBILIDAD A LA INTELIGIBILIDAD \\ Rehabilitación del sentir en Maurice Merleau-Ponty
}

\author{
Maria Carmen López Sáenz \\ UNED (Madrid) *
}

En este trabajo caracterizamos la filosofia de M. Merleau-Ponty como una fenomenología de la expresión ontológica, por considerar que reflexiona sobre algunos impensados de Husserl y los prolongá en uña nueva ontólogía contribuyendo así, una vez más, a superar los dualismos heredados, en este caso, el que se produce entre fenomenología y ontología.

Aunque haremos un recorrido por toda la obra merleau-pontiana, para pensar el sentir nos centraremos en la última, en la que el cuerpo vivido, pieza clave de la rehabilitación ontológica de lo sensible, interacciona entre lo visible y lo invisible y la percepción se redefine como proceso que ocurre entre mi carne y la carne del mundo.

Así veremos cómo Merleau-Ponty desarrolla la génesis y la síntesis pasiva explicitando el problema de la estética transcendental, implicita en Husserl. Ésta se inicia con la reflexividad corporal y culmina en la reversibilidad siempre inminente de la carne. La fenomenología describe la experiencia originaria de ese sentir desde dentro de su propia evidencia.

Ya en La estructura del comportamiento (1942), Merleau-Ponty cọnisidera el organismo biológico como significación, es decir, como orientación hacia un mundo significativo. Lo describe en relacióñ con su medio, como una "dialéctica encarnada"' que pone de manifiesto un sentido para el sújetó perrceptor. Define el comportamiento como unidad de interioridad y exterioridad, mezcla de idea y naturaleza. El comportamiento es forma (Gestalt), es decir, un todo significativo, puesto que denota la existencia en un horizonte y el sen-

\footnotetext{
- Este trabajo se realizó en el contexto del proyecto de investigación, "La filosofia ante los retos de un mundo plural. Historia, mundo de la vida e interculturalidad", subvencionado por el MEC, 2004-2007. HUM2004-03533/FISO.

I M. Merleau-Ponty, La Structure du comportement. Paris: PUF, 1942, p. 228.
} 
tido $^{2}$ resultante de la conjunción figura-fondo. En tanto forma, el comportamiento integra e interpreta los estímulos de modo que, más que de reacción del organismo, debería hablarse de expresión del mismo, hasta el punto de que si "la unidad de los sistemas físicos es una unidad de correlación, la que caracteriza a los organismos es una unidad de significación"; ${ }^{1}$ aquélla coordina mediante leyes; ésta religa en virtud del sentido; por eso el comportamiento del organismo no puede definirse como una suma de procesos, sino como el despliegue temporal y espacial de ciertas unidades ideales, como un conjunto de gestos dotados de una inteligibilidad interna a su propia organización global. Como decía Spinoza, "el todo en el organismo es una idea"2, pero en Merleau-Ponty, esa idea no procede del exterior. El comportamiento posee una estructura ideal que permite relacionar el estado actual del organismo con otro anterior y ver en él la realización progresiva de una "esencia". Esa estructura no es constituida por la conciencia, sino expresada en la existencia. ${ }^{3}$ La finitud que ésta conlleva determina la contingencia de lo vivido, que "es una amenaza perpetua para las significaciones eternas en las que aquél cree explicarse por entero". 4

Lo característico del ser humano no es tanto la capacidad de crear una segunda naturaleza, como la de superar las estructuras dadas para crear otras; este movimiento de transcendencia respecto de la significación vital de las situaciones dadas no ha de entenderse como oposición a las mismas, sino como integración de lo inferior por lo superior dotando así al primero de un nuevo significado. ${ }^{5}$ Las conductas, denominadas "superiores" dan un nuevo sentido a la vida orgánica y el espíritu requiere las actividades más simples para instituirse, de modo que no hay jerarquías, sino transcendencia, entendida como apertura inmanente al cuerpo-sujeto de comportamientos significativos y como iniciación al mundo gracias a él (être-au-monde), a su intencionalidad operante (Fungierende) dirigida a cosas que tienen "espesor sensible". 6 Por su parte, la conciencia, siempre incorporada, "puede vivir en las cosas existentes sin reflexión, abandonarse a su estructura concreta que todavía no ha sido convertida en significación expresable". ${ }^{7}$ Así es como Merleau-Ponty atestigua la

\footnotetext{
Cfr. M. Merleau-Ponty, op. cit. p. 223.

M. Merleau-Ponty, op. cit. pp. 168-169.

M. Merleau-Ponty, op. cit. p. 173.

M. Merleau-Ponty, Cfr. op. cit. p. 227.

M. Merleau-Ponty, op. cit. p. 240.
}

5 Para Merleau-Ponty, no hay reduccionismo entre los órdenes de la materia, la vida y el espíritu, sino interrelaciones y reestructuración de un orden por el que le ha precidido; así la vida es aparición de un interior en un exterior. Esta comprensión del organismo como totalidad en la que lo vital y lo espiritual en el el ser humano son interdependientes la hereda Merleau-Ponty de K. Goldstein. Véase K. Goldstein, La structure de l'organisme. Paris: Gallimard, 1951, p. 380 (Publicado en 1934). 
existencia de un mundo silencioso, pero significativo, que integra nuestras experiencias y posee significados tácitos.

Lo propio del mundo sensible es ser representativo del todo en virtud de que cada parte está imbricada en el conjunto por provenir de idénticas raíces; es decir, lo característico de lo sensible es presentar lo impresentable que es el mundo mismo como unidad. La sensibilidad comienza a adquirir un sentido ontológico, porque es ese silencioso entrelazamiento corporal con el mundo lo que posibilita la sensibilización y ésta actúa como el tercer término, siempre presupuesto, entre la materia pasiva y el espíritu activo, entre el mundo objetivado y la conciencia-sujeto.

Sin embargo, aunque en La Estructura del comportamiento, Merleau-Ponty vislumbra la significatividad de las estructuras gestálticas, no hay que olvidar que una forma es un conjunto que tiene un sentido precisamente por su capacidad de reconfiguración, pero no es una idea, si entendemos por ésta una significación eterna y aislada en su universo de inteligibles puros. En esta obra, aún late el dualismo, ya que finaliza preguntándose por la relación entre la conciencia perceptiva y la conciencia intelectual y apuntando la necesidad de integrar la filosofía transcendental en el fenómeno de lo real. Queda demostrado que éste no carece de sentido, pero el suyo se constituye en la intencionalidad de la vida comprehensiva, no en la intelección. Ciertamente, en el conocimiento puedo distinguir la "zona de las perspectivas individuales y la de las significaciones intersubjetivas", 8 pero la primera no responde a la sensibilidad y la segunda a la inteligencia, porque el horizonte de lo percibido se extiende más allá del perímetro de visión y los objetos que impresionan la retina se disponen en perspectiva alrededor del núcleo sensible. Incluso cuando pienso un triángulo no lo concibo como un ser eterno forjado desde determinados datos sensibles, sino que percibo aquí y ahora el triángulo como tal, su contenido y su forma.

El estilo de las ideas, primero tácito y después expresado, es semejante al de nuestra percepción. Ésta es la garantía de una significación ideal de lo existente que subsiste bajo la diversidad sensible. Esto puede parecer paradójico, porque solemos aplicar a la percepción la dualidad entre estructura y significación, cuando la verdad es que la Gestalt es un conjunto que tiene un sentido, pero no una idea pura, ya que se constituye y reorganiza ante nosotros como un espectáculo: "la 'cosa' natural, el organismo, el comportamiento ajeno y el propio sólo existen por su sentido, pero el sentido que brota en ellos no es aún un objeto kantiano, la vida intencional que los constituye no es una representación, la 'comprehensión' que da acceso a ellos no es todavía una intelección". ${ }^{9}$ El cuerpo comprehende el mundo sin necesidad de representárselo. 
Para la fenomenología de la existencia, "comprender" es ver las relaciones y no intentar hacer abstracción de ellas por la vía analítica o explicarlas con conceptos construidos. Merleau-Ponty está de acuerdo con Cassirer en que la relación simultánea entre materia y forma produce una "gravidez simbólica". ${ }^{10}$ Esta pregnancia descansa en la articulación de lo sensible por su estructuración perceptiva.

La conciencia perceptiva es el cuerpo vivido, que pertenece tanto al nivel natural y como al transcendental, como parte de la vida y condición de la misma, respectivamente. El núcleo de la preocupación merleau-pontiana es el movimiento por el que el ser corporal trasciende su mera materialidad hasta una existencia significativa y, a la inversa, el hecho de que todo significado se enraiza en la vida. Como ha dicho Barbaras, la fenomenología de la vida de Merleau-Ponty apuesta por un sentido que trascienda la oposición entre vida biológica y vida metafórica, ${ }^{11}$ es decir, un significado de la existencia en cada una de sus manifestaciones.

Para ello, busca la Urdoxa husserliana en el cuerpo; por eso se aleja de la intencionalidad de nuestras representaciones para centrarse en otra más originaria, la Fungierende Intentionalität, (intencionalidad operante), que encarna la unidad natural y antepredicativa del mundo con nosotros y es paralela a la unidad de los objetos experimentados, en virtud de la aprehensión precognitiva de la unidad corporal.

Frente a la intencionalidad tética del pensamiento que pone delante al objeto, la verdadera intencionalidad es pro-positiva y operante, "abierta a su objeto"12 desde el interior del ser. Se trata de una intencionalidad irreductible al pensamiento, en la que está depositado el saber que el cuerpo va acumulando; de ahí que pueda denominársele " intencionalidad corporal", ya que es el cuerpo el que la efectúa en su tendencia al mundo desde el fondo pre-reflexivo de la existencia carnal. Se identifica con la motricidad del movimiento orientado y de sus proyecciones. Esta intencionalidad vivida opera de manera latente, pasiva, cinestésica; no constituye lo que siente en su inmanencia, sino que lo toma de lo sensible sintetizándolo de una manera no representativada. Esta es la verdad por la que Merleau-Ponty se interesa y, sin embargo, no es un caso particular de conocimiento, sino una manera de adhesión y al mundo $\sin$ necesidad de pasar por las representaciones, una praktognosie ${ }^{13}$ (practognosia), una inteligencia corporal, fundamentalmente centrada en las habitualizaciones.

10 Cfr. M. Merleau-Ponty, Phénoménologie de la perception, Paris: Gallimard, 1945, p. 148.

11 Cfr. R.. Barbaras, "A Phenomenology of Life", T. Cartman, M. Hansen (eds.) The Cambridge Companion to Merleau-Ponty. Cambridge University Press, 2005, pp. 206-230, p. 211.

12 M. Merleau-Ponty, Phénoménologie de la perception, p. 509.

13 M. Merleau-Ponty, op. cit. p. 164. 
En efecto, en la adquisición del hábito, es el cuerpo el que comprende sin subsumir datos en una idea; una vez que ya tiene el hábito, el cuerpo organiza los estímulos que le vienen del exterior en relación con los problemas prácticos. Tal competencia no es un conocimiento a priori, sino una practognosia, es decir, un saber instituido progresivamente en el cuerpo, en tanto matriz de habitualidades ${ }^{14} \mathrm{o}$ acciones intencionales cuyo propósito es la acomodación a las situaciones. El hábito no es, pues, una acción involuntaria, sino una destreza que habita en los esfuerzos corporales que lo formulan; es una maestría práctica que asegura que si se dan ciertas condiciones posturales, se sigue una conducta sin necesidad de pensamiento reflexivo, casi por acoplamiento al medio. Teniendo en cuenta que el hábito no sólo refleja la capacidad de movimiento, sino que, además, desarrolla la motricidad virtual, el poder de acción y reacción que ejercemos cuando tomamos decisiones ya no ha de atribuirse a un espíritu puro, sino al arco intencional entre él y el cuerpo.

Adquirir hábitos es poder dilatar el mundo y cambiar la existencia. ${ }^{15} \mathrm{Tal}$ posibilidad aumenta nuestra comprensión y nos permite "experimentar armonía entre aquello que pretendemos y lo que viene dado, entre intención y efectuación y el cuerpo es nuestro anclaje en el mundo". ${ }^{16}$ Comprendemos una intención o un movimiento cuando el cuerpo los incorpora a su mundo carnal. De la misma manera que "el hábito no reside en el pensamiento ni en el cuerpo objetivo, sino en el cuerpo como mediador de un mundo", ${ }^{17}$ el cuerpo en el que se inscribe puede ser adiestrado, porque lleva en sí a la mente o al espíritu desafiando los dualismos.

A pesar de que la psicología solía enfatizar la conciencia en todo acto de aprendizaje, Merleau-Ponty y sus seguidores han demostrado que ésta siempre está encarnada. De ahí ha derivado la consideración de una inteligencia sentiente que se pone de manifiesto, no sólo en las habilidades motoras, sino incluso en el plano del saber. Esta inteligencia se ha denominado "corporal", por arrancar de las habitualizaciones; ${ }^{18}$ ahora bien, no se reduce a una reten-

14 El término habitus proviene del griego hexis. Consiste en una proyección de la resignificación del mundo (natural y cultural) operada por la conciencia corporal, incorporando su propia experiencia vivida, que va adquiriendo cierta generalidad en nuestras funciones corporales. Habitus es disposición adquirida o apropiación personal de un objeto o de una actitud: "la adquisición del hábito es la aprehensión de una significación, una aprehensión motora de una significación motriz" M. Merleau-Ponty, Phénoménologie de la perception, p. 51.

15 Por citar un ejemplo, la adquisición del hábito de tocar el piano, es logro progresivo de un "saber" corporal, de familiaridad o coexistencia con el instrumento mediante el reconocimiento de su fisonomía típica. Otro ejemplo podría ser la danza, e incluso determinados juegos lingüísticos -trabalenguas- que se aprenden mediante repetidos esfuerzos corporales, modificados una y otra vez hasta lograr movimientos y sentidos adecuados. M. Merleau-Ponty, Phénoménologie de la perception, p. 169

Ibidem.

18 Así lo han hecho H. Dreyfus S. Dreyfus, "The Challenge of Merleau-Ponty's Phenomenology of Embodiment for Cognitive Science", en Haber and Weiss (edrs.) Perspectives on Embodiment: The intersections of Nature and Culture. London: Routledge, 1999, p. 117). Estos 
ción pasiva de intenciones y gestos significativos, sino que también implica la capacidad de reactivarlos de modo singular, por contraste con la pasividad motora de los aprendizajes transmitidos discursivamente.

La inteligencia corporal organiza los datos mediante síntesis corporales de implicación que confieren a los objetos la unidad de la forma percibida. Estas síntesis son productivas, porque proceden de la unidad vivida del cuerpo, que es la fuente, no el efecto, de todas las experiencias. Por su parte, la unidad corporal proviene del esquema corporal, que unifica el cuerpo de una manera práctica: en sus compromisos con el mundo y no como una imagen mental retrospectiva del mismo. Esta unificación hace que conozcamos nuestro cuerpo como un estilo o actitud de un ser activo anclado en situaciones.

Frente a la visión asociacionista que la psicología ofrecía del esquema corporal, Merleau-Ponty considera que dicho esquema es lo que posibilita esa asociación de imágenes y contenidos latentes de las distintas partes del organismo, algo así como una toma de postura global de las orientaciones en el mundo intersensorial. ${ }^{19}$ Esta esquematicidad es un sistema abierto de variaciones perceptivo-motrices que integra la estructura de los horizontes protencionales y retencionales, espaciales y temporales, de las intenciones; actuando como fondo de las mismas, este esquema dinámico proporciona una unidad transespacial y transtemporal $\mathrm{y}$, sin embargo, no es una idea, puesto que no está fuera del tiempo ni del espacio.

Las interesantes descripciones merleau-pontianas de la intencionalidad motora no son meramente psicológicas, sino que también expresan, ya en sus primeras obras, la significación ontológica que va cobrando el cuerpo. De alguna manera, con el concepto de "esquema corporal", Merleau-Ponty quiere trascender, como Husserl, tanto el psicologismo escéptico, como el dualismo metafísico y reactivar de una nueva manera la tentativa kantiana de articular la sensación y la ideación. No obstante, a diferencia de Kant, el esquematismo no objetiviza; su relación con el mundo no está mediada por las representaciones, pero el esquema corporal articula el sentido gracias a su condición de simbo-

autores han mostrado que, tanto el jugador de ajedrez como el conductor experto, a través de su talento y de una práctica prolongada en un contexto corporal, son capaces de responder a situaciones sin apenas recurrir al pensamiento, al menos tal y como éste ha sido tradicionalmente comprendido como opuesto a lo corporal. En la terminología merleau-pontiana, diríamos que el jugador de ajedrez experimentado prioriza el "yo puedo" sobre el "yo pienso" y no necesita rigurosamente intentar indagar las consecuencias de cada uno de sus movimientos.

19 "El esquema corporal es postura orientada hacia determinada tarea actual o posible". M. Merleau-Ponty, Phénoménologie de la perception, p. 116. Un desarrollo más amplio del concepto en Merleau-Ponty en LÓPEZ, $\mathrm{M}^{\mathrm{a}} \mathrm{C}$., "La existencia como corporeidad y carnalidad en la filosofia de M. Merleau-Ponty", en M ${ }^{\mathrm{a}} \mathrm{C}$. López, J. Rivera de Rosales, (Coords.) El cuerpo. Perspectivas filosóficas. Madrid: UNED, 2002, pp. 179-206, especialmente, pp. 182-189 . 
lismo natura ${ }^{20}$ y raíz de todo simbolismo. El cuerpo es simbolismo primordial porque no es una estructura más, sino el patrón de toda estructura.

Decir que el cuerpo es "simbolismo" es afirmar que hay en él un pensamiento prelingüístico que no se limita a racionalizar lo latente. Obviamente, el cuerpo no es símbolo en el sentido superficial, es decir, como representación, sino en el del simbolismo expresivo de lo otro (autrui), de la existencia; el cuerpo la simboliza porque la realiza y de él arrancan todas sus virtualidades. ${ }^{21}$ Si la existencia es movimiento por el que aprendemos a asumir una situación de hecho, el cuerpo que la ejecuta es reactivación creativa del mismo. Por otro lado, el cuerpo es el simbolismo general del mundo en el que habita. Como unión de la Physis y logos que es, el cuerpo no constituye lo que siente en su inmanencia, sino que lo toma de lo sensible; es sensible a todos los objetos a los que da significación por el modo interrogativo en que los acoge y se dirige hacia ellos.

El simbolismo corporal es natural, a diferencia de los artificiales, pero éstos derivan de aquél y sólo lo superan lateralmente reinvistiéndolo desde dentro, ${ }^{22}$ es decir, consolidando la interioridad e inteligibilidad que anida en la propia estesiología. La concepción del cuerpo como simbolismo significa ver en él la simultaneidad entre lo interior y lo exterior, entre cultura y naturaleza. El cuerpo que simboliza se comprende como elemento activo de la cultura, como una naturaleza capaz de ir más allá de sí. Decir que el cuerpo es símbolo es comprenderlo situado entre la naturaleza y el lenguaje, como origen del significado y principio organizador del mismo. El cuerpo deja, entonces, de concebirse como una caída para vislumbrarse como una teleología natural.

El cuerpo es la primera producción cultural de un sujeto y las demás son prolongaciones suyas. De ahí la comparación merleau-pontiana del cuerpo con la obra de arte, ${ }^{23}$ en ambos la expresión y lo expresado son indistinguibles, algo así como el adentro del afuera y el afuera del adentro. Los sentidos del cuerpo, como los de la obra artística, no remiten a algo exterior, sino que sólo son accesibles por contacto directo, porque cuerpo y obra son textos, tejidos de significaciones vivientes. Como la obra de arte, el cuerpo se enseña a sí mismo. Este cuerpo generalizable es la sustancia de la vida estética.

${ }^{20}$ M. Merleau-Ponty, Résumés de Cours. Paris: Gallimard, 1968; p. 180. El cuerpo es un simbolismo tácito, porque funda todo simbolismo artificial, de manera que él nos abre a la verdad y a la idealidad. Tal es la conclusión de la indagación merleau-pontiana por las relaciones entre el logos del mundo sensible y el logos explícito, entre el mundo silencioso y el lenguaje.

${ }^{21}$ Puede verse desarrollada esta tesis del cuerpo vivido y su intencionalidad operante como verdadero origen del paso de lo actual a lo virtual en LÓPEZ SÁENZ, $\mathrm{M}^{\mathrm{a}} \mathrm{C}$., "Del cuerpo fenoménico como origen del noema al cuerpo de carne" (en prensa).

Cfr. M. Merleau-Ponty, La Nature. Notes de Cours du Collège de France. Paris: Seuil, 1995, p. 290.

${ }^{23}$ Cfr. M. Merleau-Ponty, Phénoménologie de la Perception, p. 176. 
En virtud de sus conductas simbólicas, el cuerpo nos abre a la verdad y a la idealidad. Ese cuerpo es encarnación de una conciencia entendida como un "yo puedo", más que como un "yo pienso". Espíritu y cuerpo son momentos aislados artificialmente de esa potencialidad corporal. Merleau-Ponty siempre piensa el cuerpo espiritualizado y el espíritu encarnado, porque no hay interioridad sin exterioridad y a la inversa. ${ }^{24} \mathrm{La}$ encarnación es lo que permite comprender el cuerpo como algo más que un organismo, como comportamiento de un sujeto; expresa la transcendencia del cuerpo respecto a su significación meramente biológica. La encarnación implica la espiritualización del cuerpo y la sensibilización del espíritu que se realiza así concretamente en el mundo. La retroalimentación del cuerpo y del espíritu se plasma en la carne, que es su medio (Medium) originario común.

El sustrato de la vida propiamente personal es el cuerpo como sujeto de una existencia prepersonal y anónima. ${ }^{25} \mathrm{El}$ cuerpo goza del movimiento de la existencia. Ahora bien, entre la existencia personal, cuyo sujeto es el yo, y la existencia prepersonal, cuyo sujeto es el cuerpo, existe una relación temporal; ambas son intermitentes y dinámicas en su coexistencia: la disminución de la intensidad de la vida personal, hace que se destaque la vida prepersonal; en cambio, las acciones parecen suprimir, en ocasiones, al organismo. Su condición subjetiva-objetiva impide reducir el cuerpo, tanto a la conciencia aislada como al discurso, porque no sólo tiene implicaciones epistemológicas y psicológicas, sino también ontológicas. El hecho de la encarnación no se vive, sin embargo, como oscilación, sino como situacionalidad del cuerpo, que es mediador del mundo para una conciencia intencional, que es ser-para-la-cosa por mediación del cuerpo. ${ }^{26}$ Por ser visible, el cuerpo es cosa; pero en tanto que ve, las cosas son una prolongación suya.Ahora bien, él no es ni el acompañante de las cosas, ni lo que las convierte en objetos, sino el campo en el que se localizan las sensaciones. Está hecho del mismo tejido que los objetos ${ }^{27}$ y por eso puede percibirlos. Su mundanización se debe a que el cuerpo envuelve una filosofía de la carne como materialidad e idealidad: no podemos cortar el cuerpo en dos diciendo "aquí el pensamiento, la conciencia; allí la materia, el objeto". Hay una profunda circularidad en el cuerpo, a eso yo lo llamo

24 "Hay un cuerpo del espíritu y un espíritu del cuerpo y un quiasmo entre ambos" (M. Merleau-Ponty, Le Visible et l'Invisible. Paris: Gallimard, 1964, p. 294). Merleau-Ponty utiliza el término "quiasmo" cuando quiere expresar que lo que hay es identidad en la diferencia. El cuerpo es esprit captif(Ibídem), es decir, natural.

25 "Mi organismo como adhesión prepersonal a la forma general del mundo, como existencia anónima y general, desempeña, por debajo de mi vida personal, el papel de un complejo innato". (M. Merleau-Ponty, Phénoménologie de la perception, p. 99). Cfr. M. Merleau-Ponty, Phénoménologie de la perception, p. 161.

27 "Mi cuerpo es la textura común de todos los objetos y es, al menos en cuanto al mundo percibido, el instrumento general de mi 'comprensión'." (MERLEAU-PONTY, M., op. cit. p. 272). 
"carne". ${ }^{28}$ En su afán de superar los dualismos, Merleau-Ponty acuña este concepto entendiéndolo, no como reunificación de cuerpo y espíritu, sino como elemento originario que está presente en todo lo vivido.

La carnalidad o generalidad que pasa por el cuerpo se traducirá también en el reconocimiento merleau-pontiano de una carne del mundo, como si éste también fuera subjetivo por comportar una invisibilidad susceptible de manifestarse; a su vez, el cuerpo propio no será ya el sujeto constituyente de lo visible, sino que en él cristalizará la visibilidad que le precede. Todo lo que hay participa del movimiento ontogenético de la reversibilidad de la carne y de su polimorfismo. Entre mi carne y la carne del mundo no sólo hay analogía; originariamente son algo común, como el fondo y la forma intercambiables e imprescindibles para que una u otra se dén. La carne es la incorporación recíproca de mi cuerpo en el mundo y del mundo en mí.

Gracias a la aplicación del cuerpo al mundo, nuestra experiencia no consistirá sólo en captar un exterior, sino también en sentir e incorporar las sensaciones. Esta sensibilidad no es constituida, sino instituida corporalmente, en la continuidad entre la cara interna y la cara externa de cada intencionalidad motriz que habita el cuerpo. La reflexión comienza en esa equivalencia del aspecto externo del cuerpo con sus ejes interiores, en la reversibilidad carnal.

En Ideas III, Husserl ya explicitaba la Fundierung del cuerpo y el alma, la interpenetración del mundo exterior y el interior. Si el alma carece de medios de efectuación y expresión cesa de ser; por su parte, el cuerpo pierde su sentido, su ser viviente se convierte en masa físico-química. Si conocemos algo del alma es gracias a la reflexividad corporal, a la capacidad de vernos y tocarnos por la cual nos aprehendemos como sujetos y objetos a la vez.

Husserl y Merleau-Ponty, toman la experiencia de lo tocante-tocado como modelo de la presencia indivisa de un ser sentiente en el seno de la vida sensorial; esta presencia se incorpora al cuerpo vivido y se despliega en cada experiencia. El modelo de la mano que toca a la vez que es tocada ejemplifica la relación entre la dimensión pasiva y la activa del cuerpo, la realidad del viviente que es a la vez masa y gesto: cada parte deja de vivirse como tal en el cuerpo absorbida por la totalidad englobante. Cada mano pretende ser la única que toca, pero se sabe tocada por la carne.

Esa reversibilidad de lo sentiente y lo sentido es análoga a la duplicación invisible característica de lo visible y representa las virtualidades de la sensibilidad en general. El cuerpo no es el responsable de este doble tocar. Él participa de la carne, en la medida en que se estructura como perceptor-percibido. No se trata de la capacidad corporal de ser un objeto percibido y un sujeto perceptor en constante oscilación, porque la mano tocada a la vez que toca no es otro objeto, sino sustancia carnal capaz de invertir la situación. Puesto que

28 M. Merleau-Ponty, "Un entretien avec Maurice Merleau-Ponty: La philosophie et la politique sont solidaires", Le Monde, 4061 (31-XII-1960), p. 9. 
no podemos tocarnos a nosotros mismos sin reconocer esta reversibilidad potencial, la conciencia de lo sentido como tocado remite a la experiencia en tanto tocante. Esto determina que la subjetividad corporal no se localice ni en la tangibilidad ni en nuestro ser tocante, sino en el entrelazamiento de ambos, en la autorreflexión.

Lo característico de esta reflexibilidad o reversibilidad, que es el sentir, es su institución y expresión simultáneas de la génesis del sentido; éste proviene del cuerpo y no de la conciencia aislada..La explicación es que el cuerpo no sólo es entendido por Merleau-Ponty como simbolismo, sino incluso como expresión primordial de lo vivido en la praxis o actividad poiética. Su operatividad instituye los signos en signos, hace que habite en ellos un sentido en virtud de su configuración. Paralelamente a su reactivación de la intencionalidad operante, Merleau-Ponty lleva a cabo una redefinición del "sentido" de manera que éste ya no se identifica con la operación de una conciencia transcendental constituyente, sino con una adherencia a ciertos contenidos sensibles, que no es obra de la conciencia ni de sus determinaciones, sino de la capacidad de reorganizar la situación. Esta conexión descubre el sentido y la idealidad en las experiencias de la carne; Merleau-Ponty pone el ejemplo de los momentos de una sonata o los fragmentos de un campo luminoso, que se adhieren uno a otro por una cohesión sin concepto análoga a la que se da entre las partes de mi cuerpo y el mundo. ${ }^{29}$

Las adherencias de lo sensible al sentido se suman al hecho de que el significado se autoconstituye dentro de un sistema estructurado diacríticamente. Dicho sistema que fabrica diferenciaciones es la carne, el vórtice ${ }^{30}$ espacializante y temporalizante. Por participación en ella, nos hallamos en un presente visible que anuncia dimensiones espacio-temporales invisibles. Nuestra apertura carnal al mundo es Urstiftung ${ }^{31}$ de un Zeitpunkt y de un Raumpunkt que inaugura un sistema diacrítico de índices espacio-temporales. El nivel pre-reflexivo es originario y está caracterizado por la coexistencia; en él, reina el presente pre-objetivo. Así es que la vida intencional arranca del tiempo vivido del cuerpo, "modelo de las matrices simbólicas, que son apertura al ser", matriz universal de todas las reflexibilidades.

El sentido previo al enfrentamiento conciencia-objeto, a la distinción entre pasividad y actividad se autoconstituye en la intencionalidad operante interna al Ser y lo hace como sistema diacríticamente diferenciado. Esta nueva concepción del sentido pone de manifiesto la transcendencia activa de la conciencia encarnada, el movimiento por el que ella se dirige al mundo proyec-

${ }^{29}$ Cfr. M. Merleau-Ponty, Le Visible et l'Invisible, p. 199.

30 Cfr. M. Merleau-Ponty, op. cit. p. 298. La carne del tiempo es la experiencia y la ideación tiene lugar en un espacio de existencia marcado por la duración.

31 Husserl considera la sedimentación como un caso de pasividad secundaria o intencionalidad latente, la Urstiftung de un punto del tiempo

32 M. Merleau-Ponty, Le Visible et l'Invisible, p. 227. 
tándose. Asimismo, revela que hay un logos del mundo sensible que después se explicita. La unidad de dicho logos no es la de la lógica, sino de la de la coexistencia. Husserl hacía referencia al "logos del mundo estético", 33 considerándolo pregnante, es decir, generalidad englobante. Merleau-Ponty lo aplica al cuerpo y a sus lejanías, que participan de una misma visibilidad. "Pregnancia empírica" -desde la óptica de Merleau-Ponty- es "definir cada ser percibido por una estructura o sistema de equivalencias alrededor del cual está dispuesto [...] Se trata de ese logos que se pronuncia silenciosamente en cada cosa sensible, en tanto que varía en torno a cierto tipo de mensaje, del que sólo podemos tener idea por participación carnal en su sentido". ${ }^{34}$ El ser de esta pregnancia es la carne o visibilidad, la cual no se reduce a lo visualizado; es el horizonte en el que vemos y que permite ver. El logos del mundo estético refuta así los dualismos cartesianos (alma-espíritu), kantianos (sentido interno-sentido externo), hegelianos (idea-sensación).

Merleau-Ponty sabe que ambos polos no existen al margen de la adherencia al cuerpo y al mundo, que no hay razón ajena al lenguaje y al logos silencioso que la precede. Por eso, traza una tercera vía entre la doxa originaria o el fenómeno del mundo y el reino de los universales y de los transcendentales: una ontología de lo sensible. El movimiento por el que se realiza esta nueva vía es doble: por un lado, espiritualización o sublimación del cuerpo y, por otro, encarnación del espíritu. Gracias a esta dinámica, la donación de sentido se efectúa como encarnación del mismo, de manera similar a la Sinngebung husserliana, en la que el aspecto físico y el ideal de la significación van unidos: "esta revelación de un sentido inmanente o naciente en un cuerpo viviente, se extiende, como veremos, a todo el mundo sensible, y nuestra mirada, advertida por la experiencia del propio cuerpo, reencontrará en todos los demás objetos, el milagro de la expresión". ${ }^{35}$ Como ha subrayado X. Escribano, "encarnación y expresión se hallan internamente vinculadas". ${ }^{36}$

Sentir y expresar responden a la fuerza de las cosas, pero también a la del deseo que invita a expresarse $\mathrm{y}$, en general, al poder de la aisthesis; ${ }^{37}$ ésta se

33 Husserl hablaba del "logos del mundo estético". Cfr. E. Husserl, Formale und Transzendentale Logik. Husserliana XVII. The Hague: Martinus Nijhoff, 1974, p. 257. Merleau-Ponty emplea esta noción para expresar esa relación de hecho entre lo sensible y lo inteligible; ese logos sería el origen de las idealizaciones o el nivel pre-reflexivo subyacente a la reflexión. M. Merleau-Ponty, Le Visible et L'Invisible, p. 261.

M. Merleau-Ponty, Phénoménologie de la perception, p. 230.

$36 \mathrm{X}$. Escribano, Sujeto encarnado y expresión creadora. Aproximación al pensamiento de Merleau-Ponty. Barcelona: Prohom., 2004, p. 101.

37 Ésta es el campo de la sensibilidad, entendida como relación estético-sensible y sensual con el mundo. En relación con ella, $\mathrm{M}^{\mathrm{a}} \mathrm{C}$. Lopez Saenz,., "El sentido de la Aisthesis en Merleau-Ponty". En J. M.Santos, P.M. Alves, A. Barata (eds.) A Fenomenologia Hoje. Associaçao Portuguesa de filosofia Fenomenológica. Lisboa: Phainomenon, 2003. Pp. 299-311. M C., López Saenz, "Pensar el arte y la aisthesis desde una fenomenología encarnada", en J. Blasco, E. Burgos, J. Solana, (eds.) Las raices de la cultura europea. Zaragoza: PUZ, 2004, pp. 547-564. 
halla tan relacionada con la poiesis ${ }^{38}$ como el sentir y la expresión del mismo. $\mathrm{Su}$ vínculo procede de la intencionalidad corporal; la expresión de ésta en el ámbito de los significados proyecta la transcendencia del espíritu (siempre encarnado) más allá de la subjetividad.

La carne merleau-pontiana, entendida como ontogénesis de lo que hay, incluido el sentido, parte de la aisthesis y se sublima en el sentir. A lo largo de este proceso, se propaga el proceso de la reactivación merleau-pontiana de la razón por la aisthesis, como conjunción activa de presencia y ausencia, de lo ordinario y lo extraordinario con objeto de ampliar la racionalidad ${ }^{39}$ más allá del cálculo de los medios y de la mera reproducción de lo fáctico, hasta dejar cabida a la creación artística, a la sensibilidad, a la imaginación, etc. que también forman parte de la racionalidad. Ésta se halla ya en la aisthesis de una manera encarnada, anudada con la sensibilidad dando lugar al conocimiento. estético, entendido como potencia ontológica de desvelamiento y expresión creadora.

La búsqueda merleau-pontiana de racionalidad en lo invisible de lo visible, no es simplemente la determinación de lo inconsciente en lo consciente. Aunque la fenomenología y el psicoanálisis se interesen por lo latente, la intencionalidad operante no es completamente inconsciente, sino una direccionalidad motora. Además la carne, de la que tal intencionalidad se nutre, no es inconsciente, sino pre-consciente, en tanto elemento que está en todo. Toda sensación es una dialéctica entre lo consciente y lo inconsciente, puesto que en ella hay intencionalidad y receptividad, ${ }^{40}$ pero el paso de la naturaleza al logos no se explica por el inconsciente, sino por el movimiento de fenomenalización mismo que es la carne, en tanto reversibilidad entre lo visible y la idea. En otras palabras, el inconsciente del sentir no es la única cara del Ser sensible, sino la complementaria de la conciencia; no se asienta ni en un conjunto de representaciones inconscientes, ni en una segunda conciencia, sino en el trasfondo de ésta. Inconsciente es la indivisibilidad del sentir, porque no es posesión intelectual de lo que es sentido, sino desposesión de nosotros mismos, apertura a lo sentido; ahora bien, necesitamos pensarlo para tomar conciencia de ello. ${ }^{41}$ Ciertamente, Merleau-Ponty valora y estudia los descubrimientos de Freud sobre el inconsciente, pero considera que su mérito estriba en haber descubierto el entrelazamiento entre "la vida anónima del cuerpo y la vida oficial de la persona"42 y haber concebido el inconsciente como la función

38 Véase la obra de I. Matos. Merleau-Ponty une pö̈etique du sensible. Toulouse: Presses Universitaires du Mirail, 2001.

39 "El cuerpo como razón y libertad encarnadas", en F. Birulés, M I. Peña (edras.) La passió per la llibertat. A passion for freedom. Ediciones de la Universidad de Barcelona, 2004, pp. 179-186. De la misma autora, "Feminismo y racionalidad ampliada" Contrastes vol. VIII (2003). Pp. 93-107.

40 Cfr. M. Merleau-Ponty, Phénoménologie de la perception, p. 247.

41 Cfr. M. Merleau-Ponty, Résumés de Cours, pp. 178-179.

42

M. Merleau-Ponty, Signes, Paris: Gallimard, 1960, p. 191. 
espiritual del cuerpo y la encarnación del espíritu. ${ }^{43}$ El Sentir es inconsciente en este sentido; consiste en abrirse a la pasividad de la comunicación, pero no es pura inconsciencia.

Nuestra actividad está ligada a nuestra pasividad como la intencionalidad generativa que habita la carne, pero el sentir no es una facultad pasiva, porque es intencional, es decir, encuentra en lo sensible una proposición existencial y es capaz de constituir una cierta identidad del objeto sin intervención de la idea, estéticamente. Esto se debe a que la carne que lo conforma no es la antítesis de la idea, sino "el paso del mundo mudo al mundo hablante" 44 o una reversibilidad doble que se manifiesta en la existencia de la "idea como una sublimación de la carne", ${ }^{45}$ de modo que sin el cuerpo y la sensibilidad no tendríamos acceso a las ideas. El paso del logos mudo al parlante ocurre por "metamorfosis" las estructuras horizónticas de lo sensible a las que se debe la infinitud del sentido. Tal metamorfosis de la carne sensible ha de entenderse como paso a una segunda visibilidad.

La carne del sentir no es, por tanto, recepción pasiva de cualidades físicas, sino que éstas se hallan revestidas de sentido. Por su parte, la sensibilidad no consiste en una capacidad interna de percibir cualidades puras e indefinibles, sino que se halla vinculada a la afectividad y motricidad. Sentir no es únicamente redibir datos externos, porque no hay frontera radical entre el dentro y el fuera, sino comunicación vital activa, ya que es la presencia del cuerpo vivido la que produce significaciones y anima las cualidades; además, el sujeto sentiente ha de poner en juego su existencia y acoger lo que le viene propuesto por el mundo sensible. En definitiva, el sentir es pasivo (respuesta) y activo (creación y génesis del sentido). Apresenta un aspecto intencional, tiene un contenido, en parte representativo, pero también operante. Para comprenderlo, tenemos que situarnos al margen de las ideas de sujeto y objeto y adentrarnos en la "capa primordial en la que nacen las ideas lo mismo que las cosas", ${ }^{47}$ la carne que contiene su propia negatividad porque es ser en dehiscencia.

Esa capa es el sentir y sus sentidos. Merece la pena recordar que ambos tienen en castellano múltiples significados que van desde lo percibido hasta lo juzgado y entendido; "sentido" es, además razón de ser, finalidad, significado $y$, por supuesto, cada uno de los cinco sentidos. Merleau-Ponty no los aborda en función del conocimiento, según la gnoseología tradicional, sino como la

43 M. Merleau-Ponty, Résumés de Cours, p. 179.

44 M. Merleau-Ponty, Le Visible et l'Invisible, p. 202.

45 M. Merleau-Ponty, op. Cit. P. 203.

4648 Así lo denomina Merleau-Ponty en las últimas páginas de Lo Visible y lo Invisible (Véase especialmente, p. 200) y lo subraya M. Carbone, The thinking of the sensible. Merleau-Ponty's A-Philosophy. Evanston: Northwestern University Press, 2004, p. 39

47 M. Merleau-Ponty, Phénoménologie de la perception. p. 254. 
expresión de una relación primordial con el mundo. Así concebidos, los sentidos se comprenden unos a otros sin tener que pasar por la idea ordenadora; el ser humano se comprende como un sensorium commun perpetuo. ${ }^{48}$ En $\mathrm{Le}$ Visible et l'Invisible, Merleau-Ponty afirma que "Cada 'sentido' es un 'mundo' i.e. absolutamente incomunicable para los otros y, sin embargo, construye algo que, por su estructura, está globalmente abierto al mundo de los otros sentidos y hace con ellos un solo Ser". ${ }^{49}$ Los sentidos son covariantes, es decir, funcionan conjuntamente sin confundirse entre sí. Derivan de uno primordial: aquél que concibe la sensibilidad como ser de dos caras, la una visible y la otra invisible. Cada uno reactiva la intencionalidad motriz del cuerpo a su manera y por eso los diferentes sentidos son reversibles, al menos virtualmente, a la vez que ofrecen una experiencia integral. ${ }^{50}$

Los sentidos se traducen directamente los unos en los otros, se comunican y simbolizan mutuamente en virtud de la unidad prelógica del cuerpo vivido instituida por el esquema corporal; ${ }^{51}$ éste funda la unidad del cuerpo propio, del sentido disperso y hasta de los objetos a los que apunta toda intencionalidad. El solapamiento natural de las partes del cuerpo, del cuerpo con las cosas y de éstas con el cuerpo hace que las sensaciones se sobrepasen siempre, sin necesidad de una idea sintetizadora de las mismas que sólo podría resultar de un pensamiento de survol. Gracias a la sinergia intencional, el cuerpo instituye el objeto visual e intersensorial, al mismo tiempo que se guía por la cosa misma, porque ésta se revela como en-sí-para-mí, pasividad y actividad.

En las últimas obras del filósofo ya no será el cuerpo el que integre los sentidos, sino la carne de la que aquéllos son diferenciaciones. Entonces, los sentidos quedarán ontologizados y despsicologizados.

Los sentidos nos inician en la significación y en la simbolización. La función simbólica de la corporeidad, a la que nos hemos referido, arranca de los gestos implicados en toda acción y expresión, los cuales trascienden el sentido físico de las situaciones y aseguran la mediación entre lo vivido y la dimensión intelectual.

En Merleau-Ponty, la expresión no se refiere únicamente al lenguaje o a sus actos, sino también a la articulación corporal que permite poner de manifiesto un sentido en génesis, expresa una manera de ser-en-el-mundo. Por sus virtualidades expresivas, captamos unitariamente el cuerpo en su doble dimensión natural y cultural sin subordinar la una a la otra. De esta manera, el gesto

48 Cfr. M. Merleau-Ponty, op. cit. p. 271.

49 M. Merleau-Ponty, Le Visible et l'Invisible, p. 271.

50 "En el sujeto normal no hay una experiencia visual, una tactil y una visual, sino una experiencia integral en la que es imposible dosificar las diferentes aportaciones sensoriales" (M. Merleau-Ponty, Phénoménologie de la perception, p. 138).

51 "En cuanto que mi cuerpo no es una suma de órganos yuxtapuestos, sino un sistema sinérgico cuyas funciones se recogen y vinculan en el movimiento general del ser-en-el-mundo, en cuanto figura estable de la existencia" (M. Merleau-Ponty, op. Cit. P. 249). 
corpóreo y.el gesto lingüístico, que sería su continuación, incorporan la capacidad significativa del cuerpo que comienza en el hecho mismo de la encarnación: "nuestro cuerpo en cuanto se mueve, eso es, en cuanto es inseparable de una visión del mundo y es la realización de esta misma visión, es la condición de posibilidad, no solamente de la síntesis geométrica, sino también de todas las operaciones expresivas y de todas las adquisiciones que constituyen el mundo cultural". ${ }^{2}$ El lenguaje corporal es gestual, natural y cultural a un tiempo, antes de la distinción entre significante y significado.

La lectura merleau-pontiana de "La pregunta por el origen de la geometría como problema histórico-intencional"33 acentúa en Merleau-Ponty el interés por la emergencia del ser ideal gracias al lenguaje, que le da carne. Según Husserl, la objetividad ideal, propia de las producciones culturales, se mantiene en la diversidad de las lenguas. Es como si éstas localizaran y temporalizaran el sentido ideal. Si éste se origina en lo sensible, si toda idea es carnal, ha de surgir de la historia. Su intemporalidad arranca justamente de su temporalidad. "Habría pues un movimiento por el cual la existencia ideal desciende en la localidad y la temporalidad, y un movimiento inverso por el cual el acto del habla aquí y ahora funda la idealidad de lo verdadero". 54

El habla tiene su origen en el gesto y es la consumación de la gestualidad corpórea, puesto que extiende hasta límites insospechados la virtualidad expresiva del cuerpo. Hay reversibilidad entre gesto y habla, ${ }^{55}$ ya que ambos son manifestaciones de la existencia cuasicarnal de la idea: "del mismo modo que la nervadura sostiene la hoja por dentro, desde el fondo de su carne, las ideas son la textura de la experiencia, su estilo, mudo al principio, proferido después". ${ }^{56}$ Toda significación está encarnada y fundamenta la idealidad en la que nos inicia la percepción. El gesto es una operación en la que lo universal se concreta y la idea se sensibiliza y también a la inversa, lo concreto perdura y lo sensible se idealiza.

En el gesto y en el habla el sentido no es un añadido a la expresión, sino inmanente a la misma. Gracias a los gestos, el cuerpo se transforma en signo y se inscribe en la lógica del sentido, que no es la de la razón analítica, sino una lógica de implicación lateral, ${ }^{57} \mathrm{o}$ del deslizamiento estructural que emana de los significados adheridos en la coexistencia sensible.

52 M. Merleau-Ponty, Phénoménologie de la perception, p. 445.

53 En 1939 aparece esta obra de Husserl en la Revue International de Philosophie con una introducción de Fink. Más tarde, el texto es incluido en Die Krisis, Husserliana VI, pp. 365-386 (Beilage III).

54 M. Merleau-Ponty, Signes, 120.

55 Sobre esta reversibilidad, de la que proceden las diferenciaciones, Véase LÓPEZ SÁENZ, $\mathrm{M}^{\mathrm{a}} \mathrm{C}$., "La Parole as a Gesture of the Originating Differentiation", En B. Penas, M ${ }^{\mathrm{a}} \mathrm{C}$. López, Interculturalism. Between Identity and Diversity. Peter Lang. (en prensa).

56 M. Merleau-Ponty, Le Visible et l'Invisible, p. 159.

57 La ontología indirecta que Merleau-Ponty propone llega al ser dialéctico a través de los entes, eliminando la diferencia ontológica. En ella, las dimensiones invisibles del ser se presentan, 
La transición de la fe perceptiva a la verdad explícita se da como paso al concepto y al mundo cultural, al mundo de las sedimentaciones. Explicitar esto es lo que Merleau-Ponty se propuso en un trabajo que no llegó a completar y que iba a llevar por título "El origen de la verdad": "hemos creído encontrar en la experiencia del mundo percibido una relación de un nuevo tipo entre el espíritu y la verdad". 58 El origen de la verdad se funda en las verdades sobre nuestros orígenes, en nuestra encarnación, que es justamente lo que nos permite salir de nosotros mismos, intentar trascender nuestros límites, percibir y hasta generar ideas.

Lo sensible y lo inteligible se unen, preservando sus diferencias, en la Carne (Chair). Las experiencias poseen idealidad porque son carnales, es decir, se adhieren al significado sin necesidad de un concepto, igual que se cohesionan las partes de mi cuerpo o éste con el mundo. Cuando se comprende que lo sensible es invisible, ya no hay problema de la idea, dice Merleau-Ponty, ${ }^{59}$ porque el concepto es primero generalidad.

El filósofo contrapone las ideas de la inteligencia o meramente adquiridas a las ideas sensibles; éstas no positivizan, sino que, desde lo sensible, de un modo aconceptual, abren a su transcendencia, porque las ideas no son entidades ocultas detrás de otras, y tampoco invisibilidades absolutas, sino "lo invisible de este mundo, lo que lo habita, lo sostiene y lo hace visible, su posibilidad interior y propia, el Ser de este ente". ${ }^{60}$ Sean inteligibles o sensibles, las ideas no pueden considerarse independientes de lo sensible, ni desligadas de las estructuras de horizonte, sino que viven de ambos, aunque su carne sea más leve, más lingüística.

Merleau-Ponty es consciente, no obstante, de que la idealidad de horizonte no agota la idealidad "pura"; intenta averiguar cómo pasamos de una a otra, por qué se agrega a la generalidad natural de mi cuerpo y del mundo otra generalidad creada, una cultura. Aunque "la creación de la cultura carece de eficacia si no encuentra un vehículo en las circunstancias exteriores", ${ }^{61}$ a pesar de que naturaleza y cultura son mutuamente dependientes e irreductibles entre sí, el mundo cultural se pone de manifiesto en el lenguaje y en las instituciones culturales; ambos nos abren a la universalidad sin abandonar lo singular. En efecto, bajo la cultura adquirida no hallamos esencias ni hechos, pero podemos encontrar la universalidad concreta de la existencia encarnada, ${ }^{62}$ si no olvidamos que el mundo cultural es la reactivación creativa del mundo percibido. Es

no como ausencia absoluta (esto no es lo que significa "invisible" en Merleau-Ponty), sino como latencia y movimiento de fenomenalización.

M. Merleau-Ponty, "Un inédit de Maurice Merleau-Ponty", Revue de Métaphysique et de Morale, 4 (1962), pp. 401-409, p. 404.

${ }_{59}$ Cfr. M. Merleau-Ponty, Le Visible et l'Invisible, p. 290.

${ }^{60}$ M. Merleau-Ponty, op. Cit. P. 198.

${ }_{61}$ M. Merleau-Ponty, Signes, p. 85.

${ }^{62}$ Cfr. M. Merleau-Ponty, Le Visible et l'Invisible, p. 162. 
como si la reprise créatice aunara lo dado con la posibilidad de su trascendencia, el sentido sensible con la transfiguración del mismo.

La dinámica de la producción de sentido en la cultura se ejerce sobre significaciones ya establecidas y signos indirectos de las mismas; éstos acaban explicitándose y dejando aún sentidos mudos que impregnan las significaciones formadas; unos y otras siguen sometidos a la lógica del sentido operante. De ahí que la cultura no proporcione significaciones transparentes, pero esto es precisamente lo que garantiza su transcendencia hacia nuevas significaciones y hacia otras culturas.

Tal transcendencia se produce en virtud del descentramiento de las significaciones sedimentadas según cada estilo particular. Éste es la verdadera definición de la idea, tal y como Merleau-Ponty la entiende, ${ }^{63}$ ya que el estilo traduce la experiencia en símbolos y cohesiona sin conceptos, dialectizando lo singular y lo universal.

Hemos visto que el estilo pertenece al cuerpo: la coordinación de todas las funciones de éste es una unidad de estilo; además, opera en la percepción organizando y estructurando los estímulos. El estilo somete, en definitiva, los datos de la experiencia a una deformación coherente del mundo prosaico sedimentado. Ahora bien, toda estilización se ejerce sobre ese mismo mundo. Merleau-Ponty llega a decir que "la sedimentación es el único modo de ser de la idealidad", 64 porque las ideas son lo invisible de lo visible, advienen como un pliegue en la pasividad y no como producción pura.

La sedimentación, de la que arranca toda idealidad, se da en virtud de nuestra Urstiftung ${ }^{65}$ espacio-temporal en el campo del Ser; esta noción no sólo muestra la pasividad de toda actividad, sino también que la sedimentación es la otra cara de la transcendencia.

La productividad de lo sedimentado en la experiencia que perdura y da sentido a nuevas experiencias será denominada por Husserl Stiftung y reinterpretada por el fenomenólogo francés como "institución", en tanto "fecundidad ilimitada de cada presente que, precisamente por ser singular y pasar, nunca podrá dejar de haber sido y, por tanto, de ser universalmente". ${ }^{66}$ Ese presente inaugura un orden, funda una institución y lo hace desde la universalidad del sentir, desde la dialéctica entre lo sedimentado y las reactivaciones individuales de las que deriva la cultura, entendida como expresión de lo interno en lo externo. Así se va forjando el orden del sentido que se autofunda en las reactivaciones expresivas de lo sensible.

63 Cfr. M. Merleau-Ponty, op. Cit. p. 160.

64 M. Merleau-Ponty, op. Cit. P. 288.

65 Merleau-Ponty pretendía sustituir la filosofia del flujo de las Erlebnisse individuales por una filosofia de la Urstiftung que arrancara del campo originario de presencia. M. Merleau-Ponty, op. Cit. p. 275. 
La teoría merleau-pontiana de la expresión, originada en el gesto, prolongada en la parole, pasando por la creación artística, hasta llegar a la expresión cultural, forma parte de la nueva ontología merleau-pontiana. En ella, la existencia convive con la esencia. Sin embargo, ésta no es, para Merleau-Ponty, una meta; si practica la reducción eidética, es porque busca su residuo, que es la existencia.

Desde la óptica merleau-pontiana, la esencia es el modo operante de comprender el mundo, pues es necesario recurrir a la idealidad para poder aprehender la facticidad: "la necesidad de pasar por las esencias no significa que la filosofia las tome por objeto, sino, todo lo contrario, que nuestra existencia está presa con demasiada intimidad en el mundo para reconocerse como tal en el momento en que se arroja al mismo, y que tiene necesidad del campo de la idealidad para conocer y conquistar su facticidad". ${ }^{67}$

No hay esencias separadas de los seres, ni intuición pura de las esencias (Wesenschau); se mezclan con los hechos en nuestra experiencia, porque el Ser al que pertenecen ya no está frente a los entes, sino que los rodea. Las esencias están encarnadas espacio-temporalmente; son lo invisible a lo que remite lo visible. Sólo aparecen en los fenómenos como idealidades en génesis. Ellos exhiben una cierta generalidad desde la singularidad de la cosa, hasta el punto de que "las cosas son Esencias al nivel de la Naturaleza". ${ }^{68}$ Cada particular encarnaría esa generalidad que le convierte en miembro de una especie. Ésta sería, por tanto, una idea sensible, pero no como resultado de una generalización inductiva ${ }^{69}$ sino como el a priori que nos inicia en la especie; ésta se da simultáneamente en cada uno de sus ejemplares y sólo se manifiesta en ellos, de modo que ofrece un conocimiento nunca clausurado. La generalidad apunta ya en las individualidades como aquello que las vincula internamente y que no existe por separado, análogamente a la especie animal que sólo es y ofrece algún conocimiento junto con los animales concretos. A su vez, éstos participan de la universalidad, en tanto variaciones o écarts de la especie.

Merleau-Ponty entiende la variación eidética, como "trabajo de la experiencia sobre la experiencia", ${ }^{70}$ que lleva a un invariante inseparable de las variaciones; es decir, no al hecho como entidad positiva separada, sino al individual estructurado por una esencia salvaje, que es la regla invisible de su generalidad. Así pues, toda esencia está adherida a la carne del mundo. No se aprehende discursivamente, sino por el método fenomenológico de visibilización intuitiva. Gracias a él, captamos la esencia como un devenir sensible efectivo; sin embargo, ésta no se construye sobre el mundo sensible, sino que lo sustenta $y$, en este sentido, funciona como una idea sensible, como un polo de la vida.

${ }^{67}$ M. Merleau-Ponty, Phénoménologie de la perception, p. IX.

68 M. Merleau-Ponty, Le Visible et l'Invisible, p. 273.

70 M. Merleau-Ponty, op. cit. p. 154 
La visibilización es la visión en medio de lo visible; no es pensamiento de ver y, puesto que, "ver es esa clase de pensamiento que no tiene necesidad de pensar para poseer la Wesen"," el pensamiento de survol positiviza las esencias; la vida, en cambio, es inherencia en el mundo; entre ambos, surge una desviación (écart) que prohibe al pensamiento proyectarse en la experiencia y "le invita a retomar la descripción más de cerca". ${ }^{72}$ Las ideas demasiado poseídas, positivizadas dejan de ser ideas, porque no dan qué pensar, no se desvían de las palabras que las expresan.

Esta visión no mecanicista de lo sensible, conduce a Merleau-Ponty a entender el pensamiento, no como lo opuesto a aquél, ni tampoco como algo introducido antes o después de lo sensible, sino como la dehiscencia de éste. Sólo hay inherencia y no pensamiento puro. Todo pensamiento es una sublimación del "hay", la realización de lo invisible, que es la potencia de lo visible, su otro lado y no su contrario. Lo que hay es lo sensible y su ser es la fenomenalización y la expresión de ese "hay", aunque éstas constituyan un proceso infinito. De la misma manera que el habla y el pensamiento se imbrican, los actos de expresión y las ideas coexisten. De no ser así, de persistir el dualismo, las ideas dejarían de ser transición de los significados disponibles a los que estamos construyendo.

La frase de Husserl "es la experiencia todavía muda lo que hay que llevar a la expresión pura de su propio sentido",73 interpela constantemente a Merleau-Ponty. No deja de interrogarse por el paso del comportamiento a la tematización, del gesto a la palabra, del sentido perceptivo al significado lingüístico. Esta persistente preocupación por llevar a la expresión lo originario demuestra que el holismo de Merleau-Ponty no es autosuficiente, sino que se sustenta en las diferenciaciones y en el intento filosófico de interrogar al mundo sensible para dilucidarlo. El paso de la experiencia muda a la verdad de la experiencia se da gracias a su expresión, a la traducción de una experiencia a su sentido. Esto requiere creación, aunque sólo sea para dinamizar los significados adquiridos, intensificarlos y desautomatizarlos. Sin embargo, la creación siempre se gesta en un fondo de sentidos sedimentados y hasta de impensados.

Merleau-Ponty cita con frecuencia a Proust para ilustrar la idea de la encarnación sensible como mediación necesaria para conocer significados espirituales. Dice que nadie como él ha sabido fijar las relaciones entre lo visible y lo invisible, describir la idea, que no es lo contrario de lo sensible, sino su envés y profundidad. ${ }^{74}$ Entre lo sensible y lo inteligible se produce una reversibilidad o reflexibidad, desde la que comprendemos la reflexión, tanto

71 M. Merleau-Ponty, Le Visible et l'Invisible, p. 301.

72 M. Merleau-Ponty, op. Cit. p. 120.

73 M. Merleau-Ponty, Phénoménologie de la perception, p. X.

74 Cfr.M. Merleau-Ponty, Le Visible et l'Invisible, p. 195. 
como la cara invisible ${ }^{75}$ de lo visible, como la exteriorización - la reflexión es $e k$-stasis ${ }^{76}$ - de un logos tácito que habita en lo sensible. Tal logos actúa en Merleau-Ponty a la manera de un cogito encarnado cuyo acto puro no es el de auto-posición, sino el de diálogo vital con su situación. Nuevamente, se hace notar la inspiración husserliana: "lo que me parece duradero, interesante, fecundo, viviente incluso hoy son los lugares donde Husserl ha subrayado la tensión entre la actitud natural y los resultados de la reflexión". ${ }^{77}$ El fenomenólogo francés se pregunta a qué puede deberse la resistencia de lo irreflexivo ante la reflexión hasta pensar que aquél subyace en ésta. Es necesario desvelar la Weltthesis pre-reflexiva, porque el papel de la fenomenología consiste en explicitar los lazos que nos unen al mundo; ${ }^{78}$ para ello, hay que interrogarse por la pasividad originaria que arranca de lo irreflexivo y actúa como trampolín de la conciencia. La reflexión remite a la conciencia intencional Fungierende, la cual no se opone al mundo sensible, sino que lo vive en virtud precisamente de su propia sensibilidad: "si el conocimiento se basa y fundamenta en el cuerpo y en la percepción vivida, no podemos sencillamente yuxtaponer de un lado la vida de la conciencia fuera de sí y de otro la conciencia de sí; la conciencia perceptiva es dialéctica sujeto-objeto"; ${ }^{79}$ en efecto, ésta no puede abstraer lo que percibe de la cosa percibida, porque es Fundierung. En esta dialéctica de la percepción el perceptor se dirige a un en-sí siempre mediado por él: "se abre a un otro absoluto que él prepara desde lo más profundo de sí". ${ }^{80}$ Percepción y pensamiento se comprenden en términos de Fundierung: la primera sería el polo fundado y el segundo el polo fundante, pero sin subordinación. Podríamos considerar la Fundierung como una hiperdialéctica de la acción recíproca, como "la reactivación perpetua del hecho y del azar por una razón que no existía antes de tal relación y mucho menos sin ella". ${ }^{81}$ De esta reactivación o Nachstiftung de la proto-institución o Urstiftung resultaría la idealidad instituyente que se exterioriza en una forma sensible.

El interés merleau-pontiano por lo que precede a la reflexión no pretende, sin embargo, quedarse en ese nivel, sino enriquecer la reflexión al comprenderla como actividad de un sujeto-objeto en el mundo y no como acción de un

75 Como buen fenomenólogo, Merleau-Ponty se atiene a lo visible, pero teniendo en cuenta el fondo invisible del que emana. Lo invisible no es constituido por el pensamiento, sino vivido como variante de nuestra corporeidad, como apariencia de comportamientos en nuestro campo de comportamiento, como la fenomenalidad o apertura a lo visible. Cuando habla de lo "invisible" no se refiere a un invisible absoluto, sino a lo que condiciona la visión, a lo que es accesible a otra visión y no como una pura nada, sino como el fondo intersubjetivo sobre el que se destaca la percepción.

76 Cfr. M. Merleau-Ponty, Le Visible et l'Invisible, p. 308.

77 AA.VV. Husserl. Cahiers de Royaumont. Paris: Editions de Minuit, 1959, p. 157.

78 Cfr. M. Merleau-Ponty, La Nature. Notes du Collège de France, p. 104.

79 M. Merleau-Ponty, La structure du comportement, pp. 227-8.

80 M. Merleau-Ponty, Phénoménologie de la perception, p. 376.

81 M. Merleau-Ponty, Phénoménologie de la perception, p. 148. 
sujeto dominador. Sólo una hiperreflexión, una reflexión ontológica y carnal consciente de la deformación que toda reflexión conlleva, podría examinar ese mundo pre-reflexivo que es su fuente. No se trata, por tanto, de poner la fe perceptiva en el lugar de la reflexión, sino de superar el pensamiento de survol; para ello, se necesita una genealogía que recupere el valor de nuestra "iniciación al mundo". ${ }^{82} \mathrm{Si}$ la reflexión remite al silencio de la percepción y éste es el hilo conductor del sentido, "se trata de crear un nuevo tipo de inteligibilidad (inteligibilidad por el mundo y el ser tal cuales, -"vertical" y no horizontal"; ${ }^{83}$ es decir, habrá que redefinir lo inteligible desde la redención de lo sensible. De este modo, lo inteligible dejará de comprenderse como supra-temporalidad y pasará a entenderse como generalidad. Ésta, como hemos visto, fundamenta la universalidad existencial del sentir, ya que integra la singularidad y la universalidad sin excluir a ninguna de ellas. ${ }^{84}$ La generalidad es interna a la individualidad, pues obedece a la generatividad de lo que hay. Merleau-Ponty retoma así la universalidad primera de la sensación de la que hablaba Husserl, la universalidad de carne y hueso, opuesta a la del concepto disociado del proceso que lo engendra desde la experiencia perceptiva. Frente a la positivización que imprime el concepto, la universalidad en curso concibe negativamente, es decir, creando intersticios que dejen ser.

De esa nueva comprensión de la universalidad como generalidad existencial proviene la universalidad sin concepto del arte, ${ }^{85}$ que se enraiza en la aconceptualidad universal del logos del mundo sensible. Esta universalidad explica que la aisthesis tenga una función de conocimiento, gracias a la cual, experimentamos la adquisición de nuevos sentidos del mundo y de nosotros mismos enriqueciéndonos y abriéndonos a la idealidad.

Idealidad y sensibilidad son campos que forzosamente se interpenetran. La última es virtualidad de las ideas y éstas, como hemos visto, son estéticas y estésicas. Merleau-Ponty se interesa por la génesis de las mismas y, por ello, su estesiología (interrogación por la génesis de la sensibilidad) ontológica se adentra en el ser de carne, entendido como lo originario que es el movimiento mismo de la vida, la transcendencia en la inmanencia por écart o divergencia: "el agente temporal=el cuerpo-el agente ideal=la palabra-Todo esto pertenece al orden de lo "transcendental" de la Lebenswelt, es decir, de las transcendencias que portan "su" objeto". " La génesis de lo transcendental es, por tanto, sensible. La reversibilidad se expande a la estesiología y la generalidad del sentiente sensibiliza toda la realidad en el sentir.

Esta devoción por lo sensible no conduce a Merleau-Ponty a concebir lo inteligible como un debilitamiento de aquél, ni a la inversa. El filósofo rede- 
fine, más bien, lo sensible, comprendido como génesis de la Carne y no como una modalidad más de la intuición. Así entendido, se excede hasta fundirse con lo invisible. Ambos pertenecen a la Carne, a su visibilidad. De ahí que "ver es siempre ver más que lo que se ve", ${ }^{87}$ puesto que es ver lo invisible en lo visible, acceder al proceso por el que algo se hace visible. No veo lo visible desde el fondo de la nada de mi conciencia, sino desde lo visible que yo soy siendo a la vez vidente. El mundo invisible de las ideas no es más que el reverso del mundo visible. De la reversibilidad entre estas dos caras de la visibilidad se deriva la infinitud de la visión, en tanto se dirige a todo lo que no es ella, incluido el pensamiento. Visión es pensamiento estético que hace sensible lo invisible.

Merleau-Ponty no propone, sin embargo, una génesis empirista del pensamiento, sino que lo entiende como visión vertical que reúne las perspectivas dispersas, de la misma manera que el tocar gobierna toda la vida táctil. Es evidente que para pensar es preciso ver y sentir, que "todo pensamiento conocido por nosotros adviene a una carne". ${ }^{88}$ Esa carne no es materia inerte, ${ }^{89}$ sino ese imbricamiento de lo visible en el vidente del que tenemos constancia cuando el cuerpo se ve viendo o se toca tocando. La idea es un sentido segundo de este tocar, un sentido figurado de la visión. Es intuitus mentis, porque la vemos con el ojo del espíritu. Merleau-Ponty recupera la definición cartesiana de la intuitus mentis, fundada en la analogía de la visión y entendida como pensamiento de un indivisible visual. Sin embargo, añade que "la visión es tele-visión, transcendencia". ${ }^{90}$ Decir que la idea es intuitus mentis significa, en Merleau-Ponty, que el ver y el pensar no son identidades, sino no-diferencias, claridades a primera vista.

La emergencia de la realidad inteligible no se explica, por tanto, recurriendo a síntesis ideales, sino a adherencias sensibles. Las ideas son puestas de manifiesto por la experiencia perceptiva y por la intencionalidad operante que preside toda orientación. Esto significa que la experiencia del mundo siempre pone al descubierto lo invisible, encarna las ideas universales. Es como si la vida se dilatara en las ideas y éstas volvieran luego a la vida ${ }^{91}$ y así aprendiéramos a sentir en toda su intensidad.

La fenomenología no se contenta, sin embargo, con descubrir cómo la idealidad se instala en los insterticios del sentir, ni siquiera con relatar el olvido de su suelo, sino que tiene, además, la osadía de pensar la génesis para ver la idealidad mundana desde la carne, "que le proporciona ejes, profundi-

87 M. Merleau-Ponty, op. cit. p. 300.

88 M. Merleau-Ponty, op. cit. p. 191.

89 En todo caso, sería una materia interiormente trabajada travaillée (Véase M. Merleau-Ponty, op. Cit. p. 193), pregnante y preñada de sentido.

91 Cfr.M. Merleau-Ponty, op. cit. p. 159. 
dad, dimensiones", ${ }^{92}$ por ser ella dimensionalidad universal. Por su parte, la intraontología manifiesta la necesidad de crear una inteligibilidad vertical, por medio del mundo y del ser; asimismo, rehabilita lo sensible y su modo alusivo de darse que es lo que lo aproxima a un pensamiento vivo.

\section{ABSTRACT}

En este trabajo caracterizamos la filosofía de M. Merleau-Ponty como una fenomenología de la expresión ontológica, por considerar que reflexiona sobre algunos impensados de Husserl y los prolonga en una nueva ontología contribuyendo así, una vez más, a superar los dualismos heredados, en este caso, el que se produce entre fenomenología y ontología. Aunque haremos un recorrido por toda la obra merleau-pontiana, para pensar el sentir nos centraremos en la última, en la que el cuerpo vivido, pieza clave de la rehabilitación ontológica de lo sensible, interacciona entre lo visible y lo invisible y la percepción se redefine como proceso que ocurre entre mi carne y la carne del mundo.

Así veremos cómo Merleau-Ponty desarrolla la génesis y la síntesis pasiva explicitando el problema de la estética transcendental, implicita en Husserl. Ésta se inicia con la reflexividad corporal y culmina en la reversibilidad siempre inminente de la carne. La fenomenología describe la experiencia originaria de ese sentir desde dentro de su propia evidencia. 
